\title{
Osteomielitis crónica supurativa en el maxilar superior: reporte de un caso clínico
}

\section{Chronic osteomyelitis suppurative in the maxilar superior: report of a clinical case}

\author{
Souza, LN*, Souza, ACRA**, de Almeida, HC***, Gómez, RS****, \\ López Alvarenga, $\mathrm{R}^{* * * * * *}$
}

\section{RESUMEN}

La Osteomielitis es una infección del tejido óseo que involucra a todas las estructuras del hueso provocada por microorganismos. El cuadro clínico se caracteriza generalmente por la presencia de fístula de drenaje, dolor intenso, inflamación, movilidad de los dientes involucrados en la zona afectada, fiebre y edema. El tratamiento consiste en medicamentoso y/o quirúrgico. En este artículo se presenta un caso de Osteomielitis crónica supurativa en el maxilar superior, que acomete a un paciente del sexo femenino de 82 años, en el cual se discuten los aspectos diagnósticos e histopatológicos como el tratamiento correspondiente del caso.

Palabras clave: Osteomielitis, Secuestros óseos, Maxilares.

\section{SUMMARY}

Osteomyelitis is an infection of bone tissue that involves all structures of the bone caused by microorganisms. The clinical picture is generally characterized by the presence of fistula drainage, severe pain, swelling, mobility of teeth involved in the affected area, fever and edema. Treatment involves medication and / or surgery. This article presents a case of chronic suppurative osteomyelitis in the maxilar superior of a female 82 years old patient, as well as a discussion of clinical and histopathological aspects and the treatment employed.

Key words: Osteomyelitis, Osseous sequestrous, Jaws.

Fecha de recepción: 17 de septiembre de 2009.

Aceptado para publicación: 2 de octubrede 2009.

* Prof. Adjunto de Cirugía Buco maxilofacial, Centro Universitario Newton Paiva. Belo Horizonte. Brasil.

** Prof. Adjunta de Anatomía de Cabeza y Cuello. Pacientes con necesidades especiales. Centro Universitario Newton Paiva. Belo Horizonte. Brasil.

*** Prof. Asistente de Prótesis Removibles. Universidad Federal de Minas Gerais. Belo Horizonte. Brasil.

**** Prof. Asociado de Patología Oral y Dental. Universidad Federal de Minas Gerais. Belo Horizonte. Brasil.

***** Licenciado en Odontología por la universidad del Centro Universitario Newton Paiva. Belo Horizonte. Brasil.

Souza, LN, Souza, ACRA, de Almeida, HC, Gómez, RS, López Alvarenga, R. Osteomielitis crónica supurativa en el maxilar superior: reporte de un caso clínico. Av. Odontoestomatol 2010; 26 (6): 295-300.

\section{INTRODUCCIÓN}

La Osteomielitis se define como una inflamación extensa del hueso, implicando a toda porción es- ponjosa, medular, cortical, periostio, vasos sanguíneos, nervios y epífisis. La inflamación puede ser aguda, subaguda o crónica y presenta un desarrollo clínico diferente según su naturaleza (1-5). 
En el caso de los maxilares se considera la infección odontógena como la causa más frecuente de osteomielitis. Se puede presentar a cualquier edad con un predominio entre los hombres. Afecta principalmente al maxilar inferior. En el maxilar superior es más raro, debido a una mayor vascularización $(3,6)$.

Su etiología es variada pero se pueden citar algunos factores tales como las infecciones bacterianas producidas por gérmenes piógenos, fundamentalmente estafilococos y en algunas ocasiones por estreptococos, neumococos y enterobacterias, hongos como actinomicetos, blastomicetos, coccidioides y criptococcus neoformans, traumatismos, agresiones químicas, electrocoagulación, irradiación, enfermedad periodontal, exodoncias, situaciones patológicas pulpares como caries y granulomas $(2,5)$.

Algunas condiciones predispuestas también favorecen la instalación de esta infección como: diabetes, anemia, leucemia, malnutrición, osteoporosis, enfermedad de Paget, displasia cemento-ósea, disosteoesclerosis, osteopetrosis, fluorosis y alcoholismo $(2,7)$.

La osteomielitis se clasifica en tres tipos: osteomielitis Supurativa (aguda y crónica), osteomielitis crónica esclerosante (difusa y focal) y osteomielitis periostitis proliferativa $(6,8)$.

La osteomielitis aguda supurativa se caracteriza por un dolor intenso, inflamación, sensibilidad, aumento de la temperatura, movilidad de los dientes involucrados en la zona de la osteomielitis y secreción de pus. La osteomielitis crónica supurativa es similar a la aguda, la deferencia entre ambas es que en la crónica, los síntomas están atenuados, se puede observar fístulas por donde salen el pus con los secuestros óseos y generalmente es asintomática ya que el proceso infeccioso junto con el área afectada esta encapsulado y separado del resto del hueso. Esta puede derivar de una aguda que no fue diagnosticada correctamente $o$ cuyo tratamiento no fue bien implementado. Radiográficamente en la fase aguda no se observan alteraciones óseas. Al cabo de tres semanas de iniciarse la infección se observa radiolucidez irregular con aumento de los espacios trabeculares $(3,6,9)$.

La osteomielitis crónica esclerosante focal es un fenómeno frecuente en pacientes jóvenes y correspon- de a una reacción focal del hueso a un estimulo inflamatorio de baja intensidad, generalmente asintomática. La lesión se descubre en exámenes radiográficos de rutina. Se localiza con mayor frecuencia en los ápices de los primeros molares inferiores y un pequeño porcentaje en los segundos molares y premolares. Radiográficamente se observa como una imagen radiopaca perfectamente definida $(3,6,10)$.

La osteomielitis crónica esclerosante difusa es similar la focal, solo que la etiología no es perfectamente conocida. Este tipo puede ocurrir a cualquier edad, pero es más frecuente en grupos de edad avanzada, parece ser que es debido a que el huésped es incapaz de controlar una infección subvirulenta del todo. La radiografía muestra una esclerosis difusa del hueso $(3,6,10,11)$.

La osteomielitis periostitis proliferativa es llamada también de Osteomielitis de Garré y se caracteriza por un espesamiento del periostio con depósito de osteoide y nueva cortical del hueso. Clínicamente se caracteriza con una tumefacción facial de ligera a intensa en la zona del maxilar inferior y la tumoración puede acompañarse de dolor moderado a intenso y trismo. En las radiografías oclusales se observa un propósito perióstico de nuevo hueso laminar que tiene un aspecto de piel de cebolla $(3,6,12)$.

El tratamiento de las osteomielitis se dirige a eliminar la fuente de infección, a través de la extracción del diente implicado, incisión y drenaje, fistulectomía, secuestrectomía, antibioticoterapia o tratamiento de endodoncia cuando las condiciones clínicas lo permitan (9). Podrá también incluirse en el tratamiento, laserterapia, termoterapia, oxígeno hiperbárico $(9,13,14)$.

El objetivo de este trabajo es la presentación de un caso clínico de osteomielitis crónica supurativa en el maxilar superior, evidenciar la importancia de un diagnóstico correcto y un tratamiento que mejore la calidad de vida de nuestros pacientes.

\section{CASO CLÍNICO}

Paciente femenino de 82 años de edad acude al Servicio de Odontología por presentar una infección crónica localizada en el borde maxilar superior iz- 
quierdo con una evolución clínica de 6 meses. Se han realizado exodoncias previas, sin mejora del cuadro clínico.

En la anamnesis el paciente relata que hace 7 años se sometió a cirugía de by-pass coronario, que padece de diabetes mellitus tipo 2 y que hace uso de una serie de medicamentos como Sinvastatina, Amlodipina, Adiro, Valsartan, Atenolol, Metformina y Glibenclamida. Ninguna cirugía previa de cuello o cabeza ni tratamiento radiante.

Al examen extraoral no se evidenció signos relevantes y la palpación de los linfonodos demostraba una situación de normalidad.

El examen intraoral evidenció la presencia de dos fístulas localizadas en la región de los dientes 2.22.3 (ausentes) y 2.4-2.5 (con movilidades). Presencia de reabsorción de la tabla ósea vestibular y drenaje de secreción purulenta por la fístula a través de una compresión en la zona (Fig. 1). Las hipótesis de diagnóstico clínico fueron infección odontógena u osteomielitis.

Fueron realizados exámenes complementarios como radiografías (Ortopantomografía y Periapicales); hemograma completo, coagulograma, glucemia (Dentro de límites normales) y evaluación del riesgo quirúrgico (ASA II).

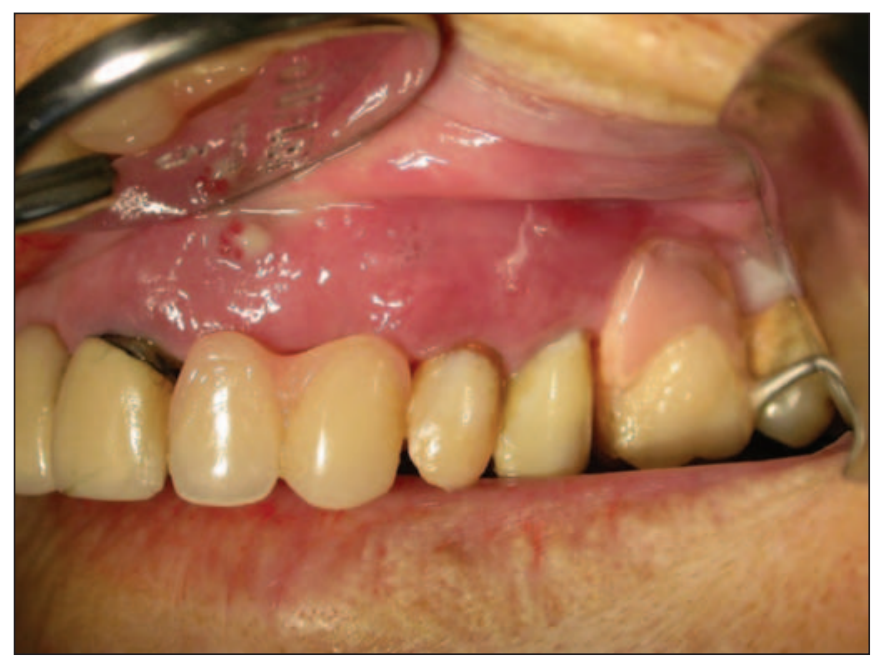

Fig. 1. Aspectos del drenaje de secreción purulenta por la fístula.
El análisis radiográfico revela una zona radiolúcida extensa de densidad alterada con presencia de secuestros óseos en la región de los dientes 2.4 y 2.5 (Fig. 2). Fue prescrito Amoxicilina (500 mg, vía oral) 1 cápsula cada 8 horas durante 14 días y cirugía con cita para después de 7 días de haber empezado el tratamiento con el antibiótico.

El tratamiento quirúrgico consistió en una curetaje, eliminación del tejido de granulación, secuestrectomía, regularización ósea, exodoncia de los dientes 2.4 y 2.5 y cierre primario de la herida quirúrgica (Fig. 3). Se remitió el material legrado en la cirugía para análisis anatomopatológico.

Resultados anatomopatológico: Los cortes histológicos coloreados con hematoxilina-eosina muestran fragmentos de mucosa revestidos por epitelio pavimentoso estratificado no queratinizado con zonas hiperplásica y exocitosis. En la lámina propia muestra tejido conjuntivo fibroso con vasos sanguíneos, edema, hemorragia y denso infiltrado inflamatorio predominantemente mononuclear. Trabéculas óseas necróticas completan el aspecto microscópico. El diagnóstico anatomopatológico fue de osteomielitis (Fig. 4).

Cuarenta días después de la cirugía, el paciente regresó con una discreta secreción en la región del

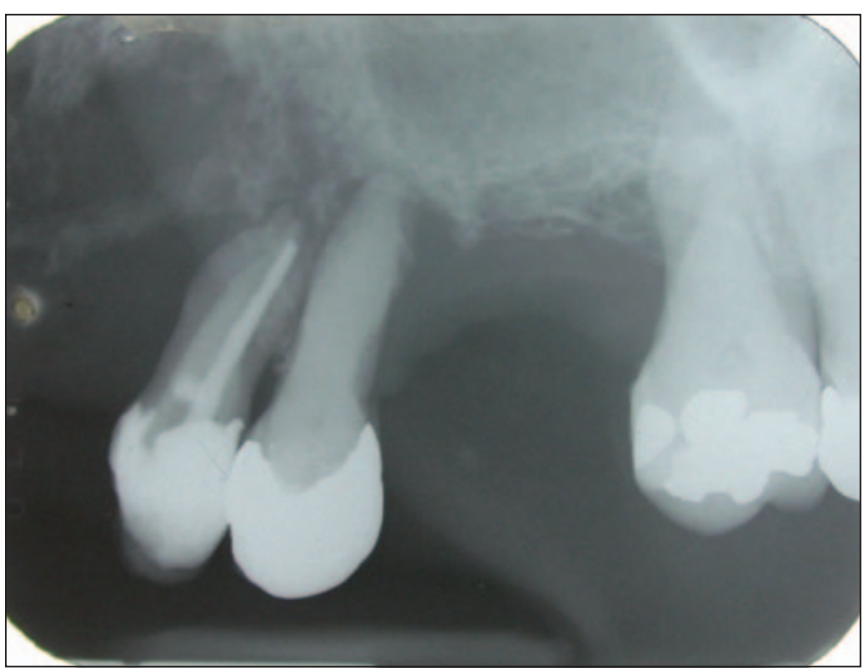

Fig. 2. Radiografía intrabucal periapical, donde se observa foco de osteomielitis con el secuestro en distintos estadios en la zona de los dientes 2.4 y 2.5 . 


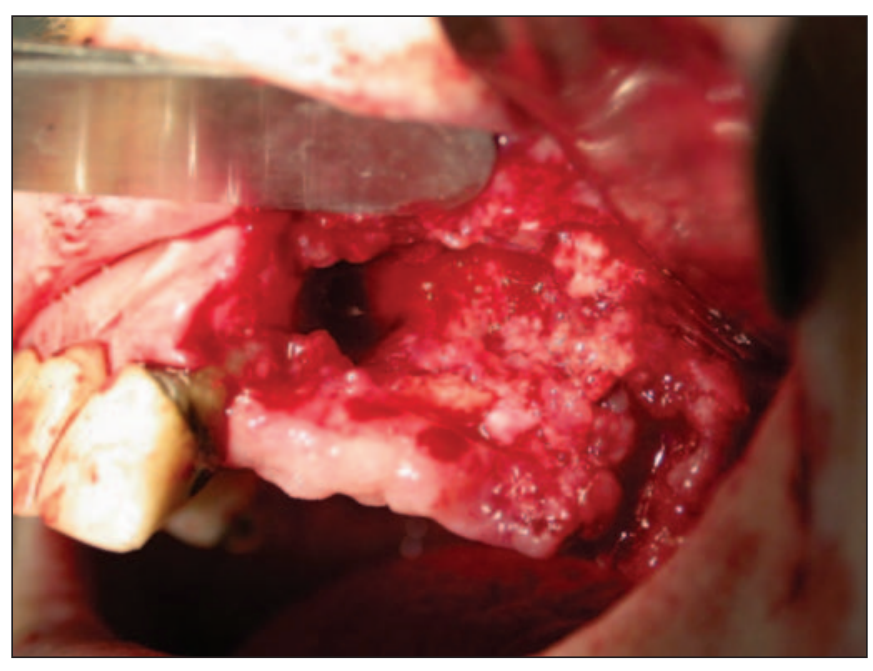

Fig. 3. Área de hueso necrótico expuesto durante el tratamiento quirúrgico.

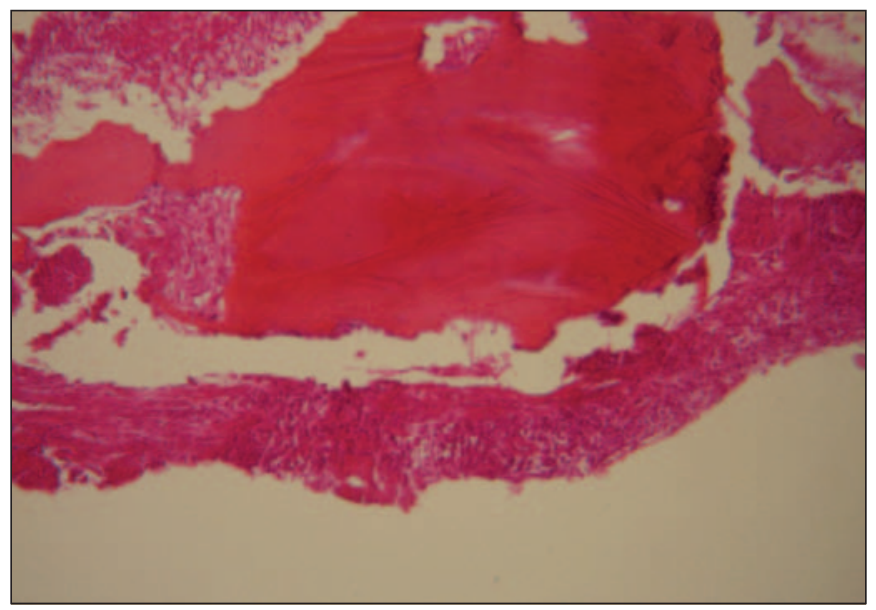

Fig. 4. Examen anatomopatológico.

diente 2.2 a través de una pequeña fístula. El examen radiográfico realizado indicó una neoformación ósea localizada. Se insistió en tratamiento con el antibiótico Clindamicina (300 mg, vía oral) 1 cápsula cada 6 horas durante 14 días, pero al séptimo día fue suspendido, debido al malestar estomacal que presentaba el paciente debido al tratamiento. La secreción purulenta estuvo prácticamente ausente, pero hubo mantenimiento de la fístula y drenaje discreta por 2 meses.

La paciente fue sometido a dos más cirugías de secuestrectomía de 5 y 6 meses desde del inicio del tra- tamiento. Se realizaron controles posteriores, observándose una evolución favorable y seis meses tras el tratamiento no hubo ningún signo de recidiva (Fig. 5).

Para el tratamiento restaurador se optó por una prótesis superior e inferior removible.

\section{DISCUSIÓN}

Prácticamente cualquier microorganismos puede infectar el hueso, pero los más frecuentes son las bacterias y las principales son estafilococos que produce cerca del $80 \%$ de las infecciones de osteomielitis $(2,5)$.

El diagnóstico se realiza teniendo en cuenta la historia clínica, examen imagenológico, análisis anatomopatológico, hemograma y leucograma con diferencial. En este caso fueron realizados también análisis de coagulograma, glucemia y evaluación del riesgo quirúrgico debido a la alteración sistémica y edad del paciente.

El análisis completo y detallado, así como la evolución del caso, permitieron confirmar el diagnóstico de osteomielitis crónica supurativa.

Los hallazgos radiográficos pueden ser tardíos o secundarios, puesto que para ser detectados debe

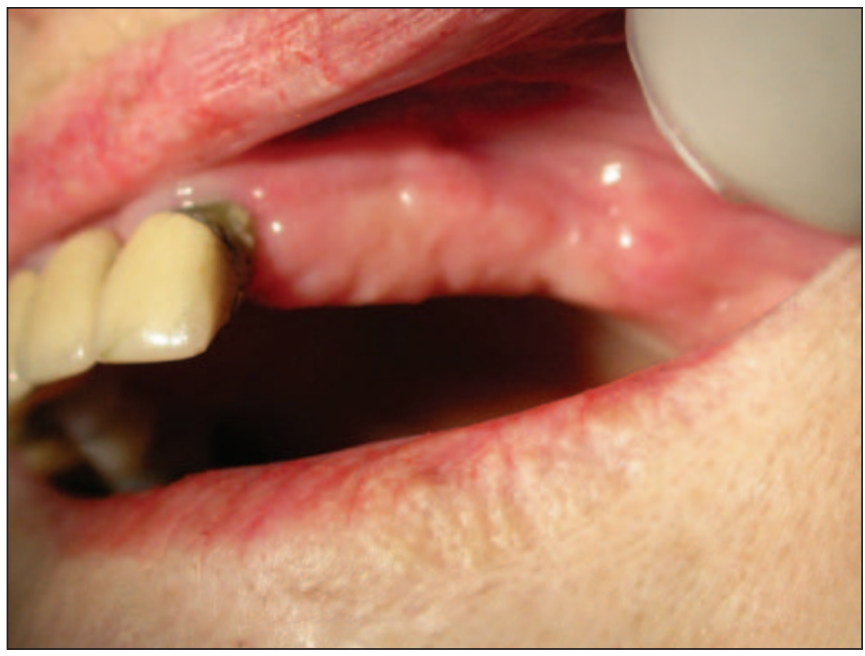

Fig. 5. Se observa cura de la sesión sobre el reborde alveolar en el postoperatorio de control. 
haberse presentado una pérdida del $30 \%$ al $60 \%$ de la porción mineral del hueso, lo cual ocurre entre 14 y 18 días después del diagnóstico clínico de la osteomielitis (9).

Las manifestaciones clínicas pueden variar según el tipo de osteomielitis. Cuando estamos frente a osteomielitis crónicas el dolor no es un signo frecuente, por la general presentan fístulas que se activan por cortos periodos de tiempos.

El tratamiento depende del estadio de la enfermedad y del control que pueda hacerse sobre el factor etiológico que la produjo. Generalmente se recomienda iniciar con las penicilinas (siempre y cuando el paciente no sea alérgico) $(14,15)$. En caso que el tratamiento con antimicrobianos no sea suficiente se recomienda tratamiento quirúrgico (incisión y drenaje, extracción del diente causal, secuestrectomía) (9). El protocolo utilizado para el tratamiento de este caso particular, fue el uso de antibióticos juntamente con una terapia de intervención quirúrgica. Los antibióticos de elección fueron amoxicilina y clindamicina, debido a sus efectos de amplio espectro, a sus acciones efectivas sobre los tejidos óseos y la no aparición de recidivas.

El diagnóstico diferencial descrito en la literatura incluye celulitis facial odontógena, abscesos o adenoflemones, inflamación de las de las glándulas salivales, hipertrofia masetérica, hiperostosis cortical infantil o Síndrome de Caffey-Silverman, Sarcoma de Ewing, displasia fibrosa, hiperparatiroidismo, enfermedad de Paget, fibroma osificante, granuloma reparador, condroma y osteoclastoma, tuberculosis, sífilis (6). Frente a un cuadro clínico como el descrito anteriormente se pensó en una infección odontógena u osteomielitis, sólo después de realizar el examen radiográfico que se pudo concluir el diagnóstico clínico. El diagnóstico definitivo se obtuvo con el examen anatomopatológico.

Con el advenimiento de los antibióticos se considera su pronóstico favorable de las osteomielitis, aunque puede entorpecerse en pacientes con enfermedades sistémicas debilitantes, alteración en la capacidad de respuesta a la infección y que se encuentra bajo tratamiento con corticosteroides.

\section{CONCLUSIONES}

La osteomielitis es una infección cuyo riesgo principal es la cronicidad con afección de la función. El diagnóstico temprano y un tratamiento correcto es la base para asegurar la recuperación y disminuir el número de recurrencias. A pesar de que su diagnóstico puede suscitar dudas, por la similitud con otras entidades, es posible su detección reuniendo todos los datos necesarios. El periodo de tratamiento puede ser largo, pero se cuenta con varias alternativas para lograr la remisión de la enfermedad, basadas, en primer lugar, en la antibioticoterapia.

\section{BIBLIOGRAFÍA}

1. Paim LB, Liphaus BL, Rocha AC, Castellanos ALZ, Silva CAA. Chronic recurrent multifocal osteomyelitis of the mandible: report of three cases. Jornal de Pediatria. 2003;79:(5): 467-70.

2. López EM, Salas AC. Osteomielitis. Criterios actuales e importancia para el estomatólogo. Rev. Cuabana Estomatol. 2001;38(1):52-66.

3. Perez C. Osteomielitis de los maxilares. Acta Odontológica de Venezuela. 1994;32(3):5-8.

4. Barry CP, Ryan CD. Osteomyelitis of the maxilla secondary to osteopetrosis: Report of a case. Oral surgery Oral Medicine Oral Pathology 2003; 95 (1):12-5.

5. Adekeye EO, Cornam J. Osteomyelitis of the jaws: a review of 141 cases. Br. Journal of Oral Surg 1985;23-44.

6. Spazzin AO, Camargo B, De Conto F, Flores ME. Osteomielite dos Maxilares. Rev. Médica HSVP. 2004;16(34):23-7.

7. Singer SR, Mupparapu M, Rinaggio J. Florid cement-osseous dysplasia and chronic diffuse osteomyelitis: Report of a simultaneous presentation and review of the literature. The journal of the American Dental Association. 2005;136 (july): 926-31. 
8. Shafer WG, Hine MK, Levy BM. A textbook of oral pathology. Philadelphia: W.B. Saunders, 1974.

9. Yeoh SC, Macmahon S, Shifter M. Chronic suppurative osteomyelitis of the mandible: Case report. Australian Dental Journal. 2005;50:(9): 200-3.

10. Van Merkesteyn JPR, Groot RH, Bras J, MCCarrol RS, Bakker DJ. Diffuse sclerosing osteomyelitis of the mandible. A new concept of its etiology. Oral Surg Oral Med Oral Pathol 1990; 70:414.

11. Marx RE, Carlson ER, Smith BR, Toraya N. Isolation of Actinomyces species and Eikenella corrodens from patients ith chronic diffuse sclerosing osteomielitis. J Oral Maxillofac. Surg 1994;52:26-33.

12. Júnior NL, Gabrielli MFR, Gabrielli MAC, Vieira EH, Barbeiro RB. Periostite Ossificante (Osteomielite de Garré) relato de caso. BCL 1997;4(3): $15-22$.
13. Van Merkesteyn JPR, Groot RH, Van Den Akker HP, Bakker DJ, Borgmeijer-Hoelen AMMJ. Treatment of chronic suppurative osteomyelitis of the mandible. International journal of oral and maxillofacial surgery. 1997;26(6):450-4.

14. Pozza DH, Neto NR, Sobrinho JBM, Santos JN, Weber JBB, De Oliveira MG. Combined treatment by antibiotic therapy and surgery of chronic mandibular osteomyelitis: a case report. R Ci Méd Biol. 2006;5(1):75-9.

15. Norden CW, Shinners E, Niederrites K. Clindamycin Treatment of experimental chronic osteomyelitis due to Staphylococcus aureus. J Infect Dis. 1986; 153:956-9.

\section{CORRESPONDENCIA}

Rodrigo López Alvarenga

Gomera, 6, Esc. 2, $3^{\circ} \mathrm{H}$

04770 Adra. Almería

E-mail: mre_lopez@hotmail.com 